\title{
Излучательная рекомбинация, захват носителей заряда на ловушки и релаксация фототока в PbSnTe:In с составом вблизи инверсии зон
}

\author{
(С) Д.В. Ищенко, И.Г. Неизвестный \\ Институт фризики полупроводников им. А.В. Ржанова \\ Сибирского отделения Российской академии наук, \\ 630090 Новосибирск, Россия \\ E-mail: ischenkod@isp.nsc.ru
}

(Получена 18 апреля 2017 г. Принята к печати 28 ноября 2017 г.)

На основании представлений о том, что PbSnTe:In является прямозонным полупроводником, выполнены расчеты времени жизни излучательной рекомбинации, а в предположении, что PbSnTe:In это неупорядоченная структура, в которой существуют центры захвата, рассчитана релаксация фототока и зависимости мгновенного времени жизни электронов и дырок. Приведенные расчеты объясняют такие наблюдаемые в эксперименте особенности PbSnTe: In, как высокая фоточувствительность в широком диапазоне длин волн, стабилизация уровня Ферми, долговременная релаксация фототока. Проведено также сравнение расчетов с экспериментальными данными и оценка возможных параметров фотоприемников.

DOI: 10.21883/FTP.2018.07.46036.8614

\section{1. Введение}

Твердый раствор $\mathrm{Pb}_{1-x} \mathrm{Sn}_{x}$ Те, легированный индием (LTT:In), является перспективным материалом для создания фотоприемников для ИК и терагерцового диапазонов. При определенном содержании олова в нем происходит инверсия зоны проводимости и валентной зоны и достигается состояние, когда ширина запрещенной зоны материала равна нулю. Вблизи этого состояния в принципе возможна регистрация фотонов с большой длиной волны. В темноте при температуре $T \leq 20 \mathrm{~K}$ для определенного содержания олова $(x \approx 0.22-0.29)$, как это следует из литературы, происходит резкое уменьшение проводимости. В работе [1] представлены результаты по созданию перехода в высокоомное состояние при увеличении содержания олова до $x=0.33$, что делает такие структуры пригодными для создания фотоприемных устройств.

Характерными особенностями LTT:In является высокая фоточувствительность - наличие реакции на крайне слабое излучение, в том числе от тел, нагретых до температур, лишь не намного превышающих температуру образцов, а также чувствительность в терагерцовой области частот [2-9]. При этом максимальная длина волны чувствительности LTT: In, зафиксированная в эксперименте, достигает 496 мкм [10]. Однако времена релаксации фототока могут достигать $10^{4} \mathrm{c}$, что может быть связано с захватом носителей заряда на ловушки [11].

Цель данной работы - расчет времени жизни излучательной рекомбинации и времени релаксации фототока в LTT: In для $x=0.29-0.33$ при захвате носителей заряда на центры прилипания, сравнение полученных расчетов с экспериментальными данными и оценка пороговых характеристик фотоприемников.

\section{2. Время жизни излучательной рекомбинации}

В работе [12] были проведены расчеты времени жизни для LTT:In c содержанием олова $x=0.24$ и было показано, что основным каналом рекомбинации при гелиевых температурах после перехода в высокоомное состояние и не слишком больших фоновых потоках является излучательная рекомбинация. Можно ожидать, что при увеличении содержания олова она останется основным каналом рекомбинации. Проведенные расчеты времени жизни излучательной рекомбинации проводились в основном в соответствии с работой Ван Русбрека и Шокли [13]. Однако, ввиду того что LTT: In является прямозонным полупроводником, значения коэффициента поглощения определялись по формуле

$$
\alpha(h v)=A\left(h v-E_{g}\right)^{1 / 2},
$$

где

$$
A \approx \frac{q^{2}\left(2 \frac{m_{h}^{*} m_{e}^{*}}{m_{h}^{*}+m_{e}^{*}}\right)^{3 / 2}}{n c h^{2} m_{e}^{*}},
$$

$n$ - показатель преломления, $c$ - скорость света, $h$ - постоянная Планка, $q$ - заряд электрона, $m_{h}^{*}$ и $m_{e}^{*}$ - эффективная масса дырки и электрона в LTT.

При изменении содержания олова эффективные массы электронов и дырок изменяются, но при сравнительно узком его изменении $(x=0.29-0.33)$, и, учитывая тот факт, что в LTT эффективные массы электронов и дырок примерно одинаковы, $A \approx \sqrt{m_{e}^{*}}$. Так как нас интересует зависимость времени жизни излучательной рекомбинации от состава, подобная точность представляется допустимой.

Время жизни носителей заряда при излучательной рекомбинации рассчитывалось для структур LTT:In c 
металлическими контактами, из которых осуществляется монополярная инжекция электронов в диэлектрик. В случае, когда концентрация инжектированных электронов много больше равновесных концентраций электронов и дырок, время жизни равно

$$
\tau_{\mathrm{ir}}=\frac{n_{i}^{2}}{R\left(n_{\mathrm{inj}}+\Delta n_{\mathrm{ph}}+n_{i}\right)},
$$

где $R$ - полная скорость излучательной рекомбинации, $n_{\mathrm{inj}}$ - концентрация электронов, создаваемая полевой инжекцией, $\Delta n_{\mathrm{ph}}$ - концентрация электронов, создаваемая оптической инжекцией, $n_{i}-$ собственная концентрация носителей заряда.

На рис. 1 представлены рассчитанные температурные зависимости времени жизни электронов для излучательной рекомбинации для LTT:In с содержанием олова $x=0.32$ (кривые $1-5$ ), а также взятые из работы [12] аналогичные рассчитанные зависимости для $x=0.24$.

Расчеты показывают, что время жизни излучательной рекомбинации уменьшается с ростом содержания олова во всем температурном диапазоне. Это связано с уменьшением ширины запрещенной зоны и ростом собственной концентрации носителей заряда.

Зависимость времени жизни излучательной рекомбинации при изменении состава в диапазоне $x=0.24-0.34$ для различных величин полевой $\left(n_{\mathrm{inj}}\right)$ и оптической $\left(\Delta n_{\mathrm{ph}}\right)$ генерации носителей при $T=4.2 \mathrm{~K}$ представлена на рис. 2.

Из рисунка видно, что время жизни излучательной рекомбинации уменьшается с ростом содержания олова в твердом растворе, причем это уменьшение зависит от уровня оптической и полевой генерации носителей заряда. В то же время наблюдается резкий экспоненциальный спад времени жизни, зависящий как от состава, так и от уровня инжекции. Этот спад (правая часть

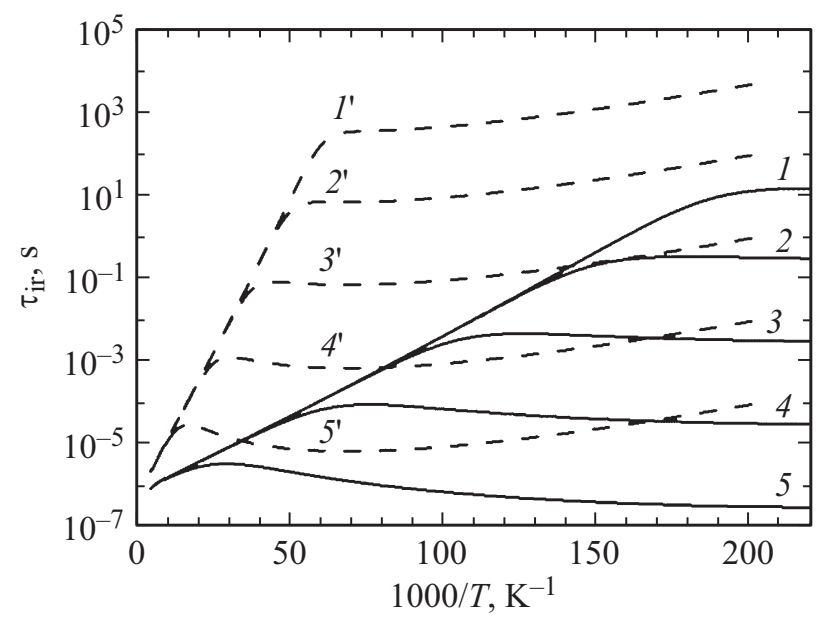

Рис. 1. Температурная зависимость времени жизни электронов излучательной рекомбинации для двух составов: $x=0.32$ (кривые $1-5$ ) и $x=0.24$ (кривые $\left.1^{\prime}-5^{\prime}\right) . \Delta n_{\mathrm{ph}}=10^{6} \mathrm{~cm}^{-3}$, $n_{\text {inj }}=10^{6} \mathrm{~cm}^{-3} \quad\left(1,1^{\prime}\right), \quad 10^{8} \mathrm{~cm}^{-3} \quad\left(2,2^{\prime}\right), \quad 10^{10} \mathrm{~cm}^{-3} \quad\left(3,3^{\prime}\right)$ $10^{12} \mathrm{~cm}^{-3}\left(4,4^{\prime}\right), 10^{14}\left(5,5^{\prime}\right) \mathrm{cm}^{-3}$.

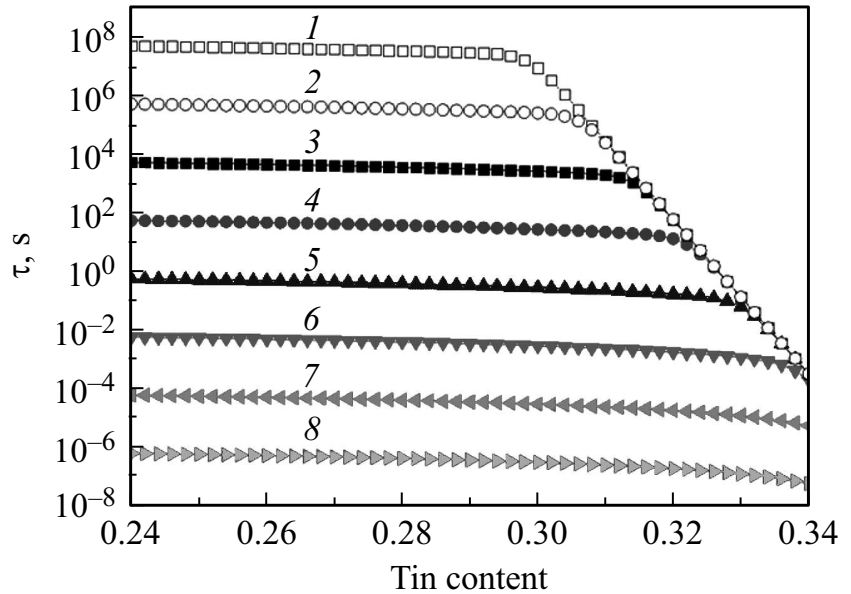

Рис. 2. Зависимость времени жизни излучательной рекомбинации от содержания олова в материале. 1 $n_{\text {inj }}=10^{2} \mathrm{~cm}^{-3}, \quad \Delta n_{\mathrm{ph}}=10^{2} \mathrm{~cm}^{-3}, 2-n_{\text {inj }}=10^{2} \mathrm{~cm}^{-3}$, $\Delta n_{\mathrm{ph}}=10^{2} \mathrm{~cm}^{-3}, \quad 3-n_{\mathrm{inj}}=10^{4} \mathrm{~cm}^{-3}, \quad \Delta n_{\mathrm{ph}}=10^{4} \mathrm{~cm}^{-3}$, $4-n_{\mathrm{inj}}=10^{6} \mathrm{~cm}^{-3}, \Delta n_{\mathrm{ph}}=10^{6} \mathrm{~cm}^{-3}, 5-n_{\mathrm{inj}}=10^{8} \mathrm{~cm}^{-3}$, $\Delta n_{\mathrm{ph}}=10^{8} \mathrm{~cm}^{-3}, \quad 6-n_{\text {inj }}=10^{10} \mathrm{~cm}^{-3}, \quad \Delta n_{\mathrm{ph}}=10^{10} \mathrm{~cm}^{-3}$, $7-n_{\text {inj }}=10^{12} \mathrm{~cm}^{-3}, \Delta n_{\mathrm{ph}}=10^{12} \mathrm{~cm}^{-3}, 8-n_{\text {inj }}=10^{14} \mathrm{~cm}^{-3}$, $\Delta n_{\mathrm{ph}}=10^{14} \mathrm{~cm}^{-3}$.

кривой 1) показывает максимально возможное время жизни для любого состава собственной концентрации носителей заряда при данных величинах оптической и полевой генерации.

Рассчитанные значения времени жизни не соответствуют экспериментально наблюдаемым временам релаксации фототока, что говорит о наличии, кроме излучательной рекомбинации, другого механизма, которым, скорее всего, является захват носителей заряда на ловушки.

\section{3. Релаксация фототока}

Для расчета времени релаксации фототока в присутствии центров захвата использовалась следующая система уравнений:

$$
\left\{\begin{array}{l}
\frac{d p}{d t}=g_{\mathrm{ph}}-\gamma_{\mathrm{ir}}\left(n p-n_{i}^{2}\right), \\
\frac{d m_{i}}{d t}=\gamma_{m i} n\left(M_{i}-m_{i}\right)-\gamma_{m i} N_{c m i} \\
\frac{d n}{d t}=\frac{d p}{d t}-\sum_{i=1}^{k} \frac{d m_{i}}{d t} .
\end{array}\right.
$$

Здесь $n, p$ - концентрации электронов и дырок в зоне проводимости и в валентной зоне соответственно, $g_{\mathrm{ph}}$ скорость оптической генерации, $\gamma_{\text {ir }}$ - коэффициент излучательной рекомбинации, $\gamma_{m i}-$ коэффициент захвата электронов на центр прилипания, $M_{i}$ - концентрация $i$-го уровня, $m_{i}$ - концентрация электронов на уровне $i$, 


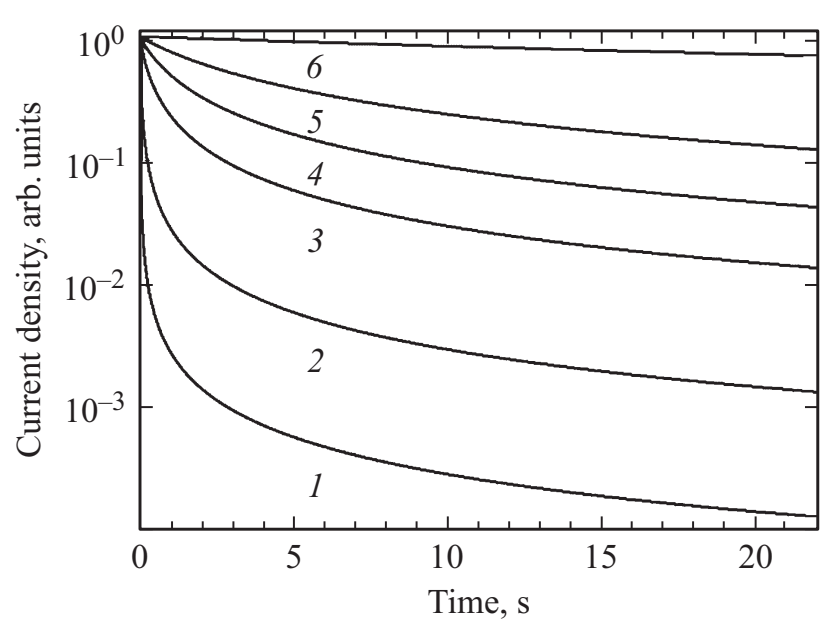

Pис. 3. Релаксация фототока для LTT:In с параметрами: $x=0.24, \quad E_{g}=0.06$ эВ $, \quad M_{1}=10^{8} \mathrm{~cm}^{-3}, \quad E_{1}=0.055$ эВ, $\gamma_{\text {ir }}=1.3 \cdot 10^{-8} \mathrm{~cm}^{3} / \mathrm{c}, \quad \gamma_{1}=2 \cdot 10^{-8} \mathrm{~cm}^{3} / \mathrm{c}, \quad g_{0}=10^{13} \quad(1)$, $10^{11}(2), 10^{9}(3), 10^{8}(4), 10^{7}(5), 10^{5} \mathrm{~cm}^{-3} \cdot \mathrm{c}^{-1}(6)$.

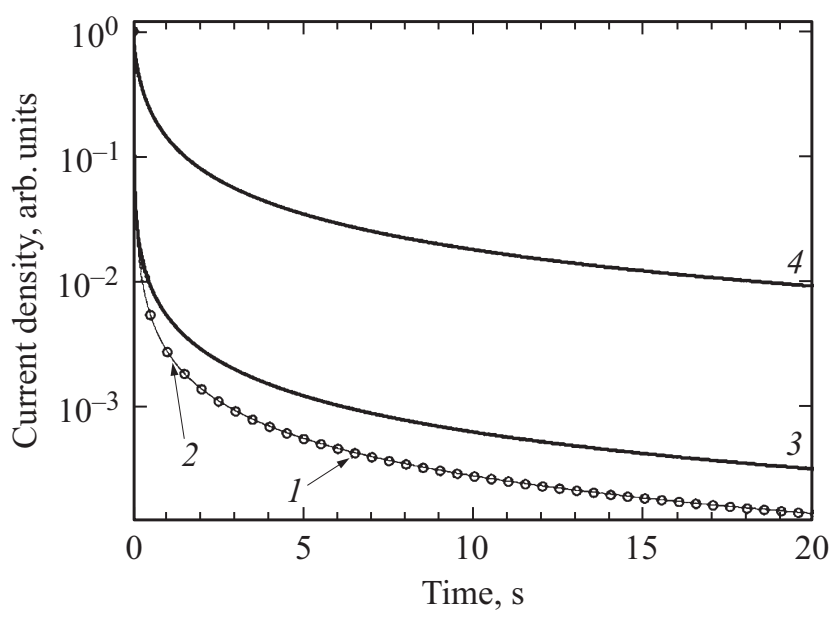

Рис. 4. Релаксация фототока для LTT:In с параметрами: $x=0.24, \quad E_{g}=0.06{ }^{\ni} \mathrm{B}, \quad E_{1}=0.055 \mathrm{\jmath} \mathrm{B}, \quad \gamma_{\mathrm{ir}}=1.3 \cdot 10^{-8} \mathrm{~cm}^{3} / \mathrm{c}$, $\gamma_{1}=2 \cdot 10^{-8} \mathrm{~cm}^{3} / \mathrm{c}, g_{0}=10^{13} \mathrm{~cm}^{-3} \cdot \mathrm{c}^{-1} . M_{1}=0$ (1, кружки), $10^{7}(2), 10^{9}(3), 10^{11} \mathrm{~cm}^{-3}(4)$.

$N_{c m}$ - эффективная плотность состояний в ЗП, приведенной к уровню $E_{i}-$ ловушек $M_{i}$, т.е.

$$
N_{c m i}=N_{c} e^{\frac{E_{i}-E_{c}}{k T}} .
$$

Расчеты проводились для случая лишь одного центра захвата носителей заряда - электронов. Это было сделано в связи с тем, что наличие даже одного центра захвата достаточно для того, чтобы объяснить основные закономерности релаксации фототока после выключения освещения.

На рис. 3 приведены расчетные кривые спада фототока от времени для LTT:In c $x=0.24$ для одного центра с параметрами, указанными в подписи к рисунку в зависимости от интенсивности оптической генерации $g_{0}$.
Из рис. 3 видно, что кинетика спада фототока существенно отличается при разной интенсивности оптической генерации. Это можно объяснить тем, что при большой интенсивности генерации все или почти все центры заполнены электронами, ток определяется только дырками и рекомбинация электронно-дырочных пар со временем жизни излучательной рекомбинации происходит очень короткое время, а затем спад фототока определяется только выбросом электронов с ловушек в зону проводимости.

На рис. 4 показаны результаты расчета спада фототока для случая, когда при одной и той же интенсивности оптической генерации изменяется концентрация центров захвата. На том же рисунке приведены результаты расчета релаксации фототока, полученные для случая излучательной рекомбинации без захвата в соответствии с формулой для спада неравновесной концентрации носителей заряда:

$$
\Delta n=\sqrt{\frac{\beta k I}{\gamma}} \frac{1}{\sqrt{\beta k I \gamma}+1} .
$$

Из рис. 4 видно, что спад фототока после выключения освещения, рассчитанный по приведенной выше формуле, практически совпадает с кривой при наличии уровня с концентрацией $M_{1}=10^{7} \mathrm{~cm}^{-3}$, полученной численным расчетом. При бо́льших значениях концентрации центров спад фототока становится все более затянутым.

При наличии захвата времена жизни электронов и дырок становятся различными. В этом случае для описания реальной ситуации полезно ввести „мгновенное“ время жизни электронов и дырок, определяемое как

$$
\tau_{n}=-\frac{\Delta n}{\frac{d}{d t}(\Delta n)} \quad \text { и } \quad \tau_{p}=-\frac{\Delta p}{\frac{d}{d t}(\Delta p)} .
$$

На рис. 5 приведена зависимость мгновенных времен жизни электронов и дырок после выключения

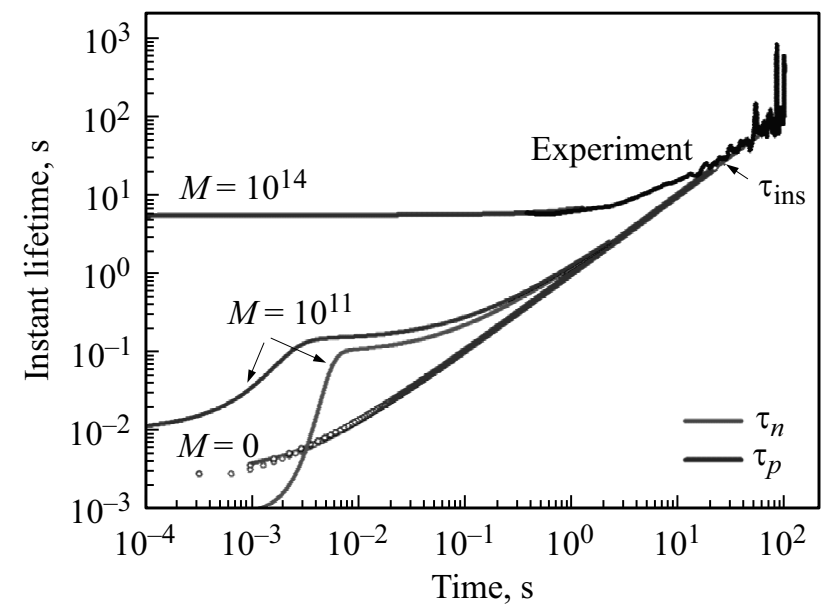

Рис. 5. Зависимость мгновенного времени жизни электронов и дырок от времени с момента выключения оптической генерации: $x=0.32, E_{g}=0.016$ эВ, $E_{1}=0.012$ эВ, $\gamma_{\text {ir }}=1.3 \cdot 10^{-8} \mathrm{~cm}^{3} / \mathrm{c}, \gamma_{1}=2 \cdot 10^{-8} \mathrm{~cm}^{3} / \mathrm{c}, g_{0}=10^{13} \mathrm{~cm}^{-3} \cdot \mathrm{c}^{-1}$. 
освещения и приведено сравнение расчетных данных с экспериментом. В данном случае расчет проводился для следующих параметров: $x=0.32, E_{g}=0.016$ эВ; $E_{1}=0.012$ эВ $, \gamma_{\text {ir }}=1.3 \cdot 10^{-8} \mathrm{~cm}^{3} / \mathrm{c}, \quad \gamma_{1}=2 \cdot 10^{-8} \mathrm{~cm}^{3} / \mathrm{c}$, $g_{0}=10^{13} \mathrm{~cm}^{-3} \cdot \mathrm{c}^{-1}$. Экспериментальные данные приводятся для образца с $x \approx 0.32$, релаксационные характеристики тока которого были приведены в работе [1]. Темно-серые линии - дырки, светло-серые - электроны, $\tau_{\text {ins }}$ - мгновенное время жизни, черным - время жизни, полученное из эксперимента.

Как и следовало ожидать, для малых значений концентрации центров захвата мгновенные времена жизни электронов и дырок совпадают. По мере увеличения концентрации центров из-за захвата электронов их мгновенное значение времени жизни становится меньше мгновенного времени жизни дырок. По мере выброса электронов с центров захвата и их рекомбинации с дырками их мгновенные времена в конечном итоге совпадают.

Фотоэлектрические свойства образцов исследовались при воздействии излучения абсолютно черного тела (АЧТ), нагретого до температур $15<T_{\mathrm{BB}}<90 \mathrm{~K}$. Ампер-ваттная чувствительность образца, находящегося при $T=4.2 \mathrm{~K}$, росла с уменьшением температуры АЧТ от $10^{2} \mathrm{~A} / \mathrm{B}$ при $T_{\mathrm{BB}}=90 \mathrm{~K}$ до $5 \cdot 10^{4}-1 \cdot 10^{5} \mathrm{~A} / \mathrm{B}$ при $T_{\mathrm{BB}}=15-20 \mathrm{~K}$. Обнаружительная способность, измеренная на частоте около 1 кГц, составляла примерно $10^{16} \mathrm{cмГц}^{1 / 2} \mathrm{BT}^{-1}$ при $T_{\mathrm{BB}}=20 \mathrm{~K}$. Как показали расчеты, это увеличение может быть связано не только с межзонными переходами, но и переходами электронов с центров захвата в зону проводимости.

\section{4. Обсуждение результатов и заключение}

Результаты проведенных расчетов для LTT:In в условиях монополярной инжекции дают возможность сделать следующие выводы.

- Время жизни излучательной рекомбинации $\tau_{\text {ir }}$ при увеличении содержания олова зависит от уровня полевой инжекции (напряжения, приложенного к образцу).

- При минимальном уровне инжекции с увеличением содержания олова $\tau_{\text {ir }}$ уменьшается в соответствии с ростом собственной концентрации носителей заряда.

- Наличие центров захвата одного сорта носителей заряда (например, электронов) приводит к увеличению мгновенного времени жизни дырок и уменьшению его для электронов и к увеличению времени релаксации фототока после прекращения освещения.

- Релаксация фототока зависит от соотношения между уровнем оптической генерации и концентрацией центров захвата.

Таким образом, введение в модель LTT:In в условиях монополярной инжекции центров захвата позволяет с единой позиции объяснить наблюдавшиеся в этом материале такие фотоэлектрические явления, как чувстви- тельность в широкой области спектра от инфракрасной до терагерцовой $[2-10,12,14]$ и долговременную релаксацию фототока $[11,15]$.

Авторы выражают искреннюю благодарность В.Н. Шумскому за помощь в проведении расчетов, обсуждении результатов и за сделанные замечания.

Работа выполнена при поддержке РФФИ, грант № 17-02-00575.

\section{Список литературы}

[1] А.Н. Акимов, Д.В. Ищенко, А.Э. Климов, И.Г. Неизвестный, Н.С. Пащин, В.Н. Шерстякова, В.Н. Шумский. Микроэлектроника, 42 (2), 83 (2013).

[2] A.E. Klimov, V.N. Shumsky. Proc. SPIE, 5964, 95 (2005).

[3] D.R. Khokhlov, I.I. Ivanchik, S.N. Raines, D.M. Watson, J.L. Pipher. Appl. Phys. Lett., 76 (20), 2835 (2000).

[4] A. Klimov, V. Kubarev, V. Shumsky. Abstract Book of the 8th Russia-CIS-Baltic-Japan Symp. on Ferroelectricity (Tsukuba, Japan, 2006) p. 63.

[5] А.Н. Акимов, В.Г. Ерков, В.В. Кубарев, Е.Л. Молодцова, А.Э. Климов, В.Н. Шумский. ФТП, 40 (2), 169 (2005).

[6] A.E. Klimov, V.V. Kubarev, V.N. Shumsky. Ferroelectrics, 347, 111 (2007).

[7] А.Н. Акимов, А.Э. Климов, И.Г. Неизвестный, В.Н. Шумский, В.В. Кубарев, О.В. Смолин, Е.В. Сусов. Прикл. физика, 6, 12 (2007).

[8] А.Н. Акимов, А.В. Беленчук, В.Г. Ерков, А.Э. Климов, И.Г. Неизвестный, О.М. Шаповал, В.Н. Шумский. Поверхность. Рентгеновские, синхротронные и нейтронные исследования, 12, 18 (2007).

[9] A.E. Klimov, V.N. Shumsky. Proc. Int. Conf. on Infrared, Millimeter and Terahertz Wave (Busan, Korea, 2009) p. 316.

[10] А.В. Галеева, Л.И. Рябова, А.В. Никорич, С.Д. Ганичев, С.Н. Данилов, В.В. Бельков, Д.Р. Хохлов. Письма ЖЭТФ, 91 (1), 37 (2010).

[11] Б.М. Вул, И.Д. Воронова, Г.А. Калюжная, Т.С. Мамедов, Т.Ш. Рагимова. Письма ЖЭТФ, 29 (1), 21 (1979).

[12] А.Э. Климов, В.Н. Шумский. ФТП, 42 (2), 147 (2008).

[13] W. van Roosbroeck, W. Shockley. Phys. Rev., 94 (6), 1558 (1954).

[14] Л.И. Рябова, Д.Р. Хохлов. УФН, 184, 1033 (2014).

[15] Б.А. Вул, И.Д. Воронова, С.П. Гришечкина, Т.Ш. Рагимова. Письма ЖЭТФ, 33 (6), 346 (1981).

Редактор А.Н. Смирнов 
The radiative recombination, the capture of carriers in the traps and photocurrent relaxation in PbSnTe: In with composition near the band inversion

\author{
D.V. Ishchenko, I.G. Neizvestny
}

Rzhanov Institute of Semiconductor Physics, Siberian branch Russian Academy of Science, 630090 Novosibirsk, Russia

Abstract In this work the lifetime of radiative recombination was calculated on the bases of PbSnTe:In being direct-gap semiconductor. Calculations of relaxation of photocurrent and dependences of instantaneous lifetime of electrons and holes were performed assuming that $\mathrm{PbSnTe}: \mathrm{In}$ is a disordered system. Calculations presented explain experimental properties of PbSnTe:In including photosensitivity in wide range of wavelengths, pinning of Fermi level and longtime photocurrent relaxation. Experimental data were compared with calculated ones and prospective parameters of photodetectors were estimated as well. 